\section{Acesso e uso de serviços de saúde em idosos residentes em áreas rurais, Brasil, 1998 e 2003}

\author{
Access to and use of health services by rural \\ elderly, Brazil, 1998 and 2003
}

${ }_{1}^{1}$ Centro de Informação Científica e Tecnológica, Fundação Oswaldo Cruz, Rio de Janeiro, Brasil.

Correspondência C. Travassos Departamento de Informações em Saúde, Centro de Informação Científica e Tecnológica, Fundação Oswaldo Cruz. Av. Brasil 4365, Rio de Janeiro, $R J$ 21040-900, Brasil. claudia@cict.fiocruz.br

\begin{abstract}
We analyzed access to health services and the utilization of such services by elderly rural residents in Brazil in 2003, comparing the patterns to those of the urban elderly and the equivalent rural pattern in 1998, using data from the $\mathrm{Na}$ tional Household Sample Survey. Access barriers were greater in rural as compared to urban areas. Health services utilization was less than in the urban elderly, even for rural elders who reported health problems. There was no difference in hospitalization rates among rural and urban elderly. Analysis of the health services that were used showed that there was limited access to services with intermediate complexity. The results suggest that access barriers increase even further with advancing age. Gender differences in utilization, generally favoring women, are more marked in the rural elderly. Financial barriers are also more evident. The health services supply should be expanded and adapted to the territorial, cultural, and social characteristics of the rural elderly.
\end{abstract}

Aged; Health Services Accessibility; Health Services Research; Rural Health
Claudia Travassos 1

Francisco Viacava 1

\section{Introdução}

O Sistema Único de Saúde (SUS) orienta-se pelo princípio da universalidade no acesso aos serviços de saúde. O Plano de Ação sobre o Envelhecimento da 2a Assembléia Mundial sobre o Envelhecimento destacou a eqüidade no acesso aos cuidados de saúde e as ações de promoção à saúde e prevenção de doenças como bases para um envelhecimento saudável 1 .

Acesso indica o grau de facilidade ou dificuldade com que as pessoas obtêm serviços de saúde 2 . $\mathrm{O}$ acesso reflete as características do sistema de saúde, que atuam aumentando ou diminuindo obstáculos à obtenção de serviços pela população. A utilização de serviços de saúde, entendida como a entrada nos serviços, é uma expressão positiva do acesso. No entanto, a utilização é influenciada também por fatores dos indivíduos, em particular, pelo perfil de necessidades de saúde e pelos valores e preferências das pessoas.

O envelhecimento associa-se a uma maior prevalência de doenças e mais incapacidades. Por essa razão, caracteriza-se por ser uma fase da vida na qual a utilização de serviços de saúde tende a aumentar. O padrão geral de utilização é marcado pelo maior uso no primeiro ano de vida e nos idosos, etapas da vida de maior vulnerabilidade biológica. Dados do suplemento sobre Acesso e Utilização de Serviços de Saúde da Pesquisa Nacional por Amostra de Domicílios 
(PNAD) de $1998{ }^{3}$ confirmam esse padrão na população brasileira ${ }^{4}$ e destacam a maior participação dos idosos no consumo de serviços de saúde 5 . O acelerado envelhecimento da população brasileira coloca grandes demandas ao sistema de saúde, marcadas pelo aumento de problemas de saúde de longa duração e pela utilização de procedimentos terapêuticos de alto custo 6 .

De modo geral, observa-se menor acesso e conseqüente menor utilização de serviços de saúde nas populações rurais 7,8. Os idosos residentes em áreas rurais, além de enfrentar os problemas de saúde do envelhecimento, têm de lidar com maiores barreiras para obter os serviços de saúde de que necessitam. Uma barreira importante enfrentada por esse grupo populacional ocorre em função da menor disponibilidade de serviços, particularmente em áreas esparsamente povoadas. Grandes distâncias a serem percorridas, dificuldades de transporte e baixa renda são fatores que, associados, reduzem a utilização de serviços de saúde ${ }^{9}$. No entanto, não foram encontrados estudos específicos sobre acesso e utilização de serviços de saúde na população idosa brasileira residente em áreas rurais.

A diversidade que define cada um dos pólos da dualidade rural/urbano tem motivado um grande debate no campo da sociologia rural no Brasil 10. Para alguns autores, dada a força com que ocorre o processo de urbanização, a aproximação desses pólos tende a representar o fim da realidade rural pelo esvaziamento do extremo rural, visto como o pólo mais atrasado. Uma segunda vertente, no entanto, considera que essa aproximação não destrói as particularidades de cada pólo, mesmo quando desaparecem as diferenças no acesso aos bens materiais e de serviços, como já ocorre em algumas áreas mais desenvolvidas do país 11.

Este estudo avalia o padrão de acesso e utilização de serviços de saúde dos idosos residentes em áreas rurais no Brasil no ano de 2003 e compara com o padrão observado na população idosa urbana. Analisa também o desempenho do sistema de saúde a partir da avaliação da ocorrência de variação no acesso e na utilização de serviços de saúde em relação ao ano de 1998, com base nos dados dos suplementos sobre Acesso e Utilização de Serviços de Saúde da PNAD 3,12.

\section{Metodologia}

Realizou-se estudo de corte transversal para avaliar o padrão de acesso e utilização de serviços entre os idosos residentes nas áreas rurais do país. Empregaram-se dados do suplemento sobre Acesso e Utilização de Serviços de Saúde da
PNAD dos anos de 19983 e 2003 12. A amostra da PNAD é representativa da população residente em domicílios particulares no país. Trata-se de amostra estratificada com até três estágios de seleção em cada estrato ${ }^{13}$. É importante destacar que em 1998 e 2003 as amostras não incluíram a população residente na área rural da Região Norte.

Foram selecionadas para estudo as pessoas com 60 anos ou mais. Os residentes em áreas rurais foram definidos pelas pessoas cujo domicílio estava situado em setores censitários classificados como: "cidade ou vila, área não urbanizada", "aglomerado rural isolado, povoado", "aglomerado rural isolado, núcleo", "aglomerado rural isolado, outros aglomerados" ou "zona rural, exclusive aglomerado rural”. No Brasil, a legislação define como rurais as áreas situadas fora dos limites das cidades (sedes dos municípios), das vilas (sedes distritais) e das áreas urbanas isoladas. A classificação adotada neste estudo se distingue da classificação do Instituto Brasileiro de Geografia e Estatística (IBGE) 14 por incluir na população rural as pessoas residentes em "área não-urbanizada de cidade ou vila" (tipo 2) e excluir as pessoas classificadas como residentes em "aglomerado rural de extensão urbana” (tipo 4).

Para avaliar as necessidades de saúde, utilizaram-se três indicadores relacionados à morbidade referida: prevalência de pessoas que auto-avaliaram seu estado de saúde como ruim ou muito ruim 15; prevalência de pessoas que declararam ter tido restrição de atividade por motivo de saúde nos 15 dias que antecederam a entrevista; e prevalência de pessoas que referiram incapacidade funcional física moderada (andar cerca de 100 metros) 16. A medida de incapacidade funcional incluiu as respostas positivas nas categorias "não consegue", "tem grande dificuldade" e "tem pequena dificuldade”, existentes no questionário da PNAD.

O acesso aos serviços de saúde foi medido com base nos seguintes indicadores: proporção de pessoas que realizou consulta médica no último ano; proporção de pessoas, entre as que consultaram, que realizou mais de uma consulta médica no período - como indicador proxy da continuidade do cuidado; proporção de pessoas que foi ao dentista no último ano; proporção de pessoas que referiu ter serviço de uso regular; tipo de serviço de uso regular. Mediu-se também a cobertura de plano de saúde público ou privado e a cobertura de mamografia nas mulheres de 60 a 69 anos, dada pela proporção de mulheres, neste grupo etário, que realizou este exame nos dois anos que antecederam a data da entrevista 17.

O indicador de utilização de serviços de saúde foi a taxa medida pelo número de idosos 
que utilizou serviços de saúde nos 15 dias que antecederam a entrevista por 100 idosos na população. Esse indicador engloba utilização dos seguintes tipos de serviço: farmácia, posto ou centro de saúde, consultório médico particular, consultório odontológico, consultórios de outros profissionais de saúde (fonoaudiólogos, psicólogos etc.), ambulatório/consultório de empresa/sindicato, ambulatório/consultório de clínica, pronto-socorro/emergência, hospital, laboratório/clínica para exames complementares e atendimento domiciliar. Outro indicador foi a taxa de internação hospitalar nos 12 meses que antecederam a entrevista, medida pelo número de idosos que se internou no último ano por 100 idosos na população. Nos casos de utilização de serviços, foi analisada a fonte de financiamento do cuidado prestado, a partir da participação no pagamento das seguintes fontes: SUS, plano privado de saúde e pagamento do próprio bolso.

$\mathrm{Na}$ análise, os indicadores foram desagregados por idade - agrupada em três categorias: 60 a 69 anos, 70 a 79 anos, 80 anos e mais - e por sexo. Compararam-se as populações de idosos residentes em área rurais e urbanas no ano de 2003 e os idosos residentes em áreas rurais entre 1998 e 2003.

Os cálculos dos indicadores e respectivos intervalos de confiança de $95 \%$ foram realizados empregando-se o programa estatístico SPSS versão 13 (SPSS Inc., Chicago, Estados Unidos), com a função analyze complex samples, que corrige o efeito de desenho da amostra. O uso de probabilidades diferenciadas na seleção da amostra implicou necessidade de ponderar as observações. Foram utilizados pesos normalizados 18 .

\section{Resultados}

Em 1998 e 2003, o tamanho da população rural idosa na amostra da PNAD foi de 6.366 e 6.220 idosos, respectivamente. A distribuição etária manteve-se estável, com $56 \%$ dos idosos no grupo etário de 60 a 69 anos e 13\% no grupo de 80 anos e mais. Não se observou variação de gênero, com homens e mulheres distribuindo-se igualmente entre os grupos etários nos dois anos analisados.

A população de idosos residentes em áreas urbanas na amostra da PNAD em 2003 é constituída por 30.776 pessoas. Sua distribuição é semelhante à que ocorre na população rural, em que a primeira faixa etária concentra $55 \%$ dos idosos e apenas $12 \%$ situam-se no grupo etário de 80 ou mais anos de idade. As diferenças de gênero entre idosos urbanos são mais evidentes, observando-se maior proporção de homens na primeira faixa de idade e maior proporção de mulheres na última faixa etária.

A avaliação do perfil de necessidades de saúde da população idosa residente em áreas rurais em 2003, dada pela prevalência de pessoas que auto-avaliaram seu estado de saúde como ruim ou muito ruim, foi de $16,6 \%$ (IC: $15,3-18,0$ ). Essa prevalência aumentou expressivamente com a idade em ambos os sexos. No grupo etário de 80 anos e mais, foi aproximadamente duas vezes mais alta, comparativamente ao grupo de 60 a 69 anos, em ambos os sexos. As mulheres idosas residentes em áreas rurais entre 60 e 79 anos de idade apresentaram prevalência de auto-avaliação ruim e muito ruim mais alta do que a dos homens. No entanto, entre os mais idosos (80 anos e mais) não houve diferença de gênero na auto-avaliação (Tabela 1). Nos idosos residentes em áreas urbanas, essa prevalência foi mais baixa: 12,9\% (IC: 12,4-13,5) deles avaliaram sua saúde como ruim ou muito ruim.

Aproximadamente $13 \%$ dos idosos rurais referiram restrição de atividade por motivo de saúde nos 15 dias que antecederam a entrevista. A prevalência aumentou com a idade, mas, em geral, não variou entre homens e mulheres (Tabela 1). Os idosos que vivem em áreas urbanas apresentaram prevalência muito semelhante (13,3 por 100 idosos; IC: 12,8-13,8).

A prevalência de incapacidade funcional moderada (21,4\%; IC: 20,0-22,8) aumentou expressivamente com a idade nos idosos rurais em 2003. Embora essa prevalência fosse maior para as mulheres em todas as idades, o aumento com a idade foi relativamente maior no caso dos homens (Tabela 1). O comportamento foi muito semelhante nos idosos residentes em áreas urbanas e rurais.

Em 2003, seguindo o padrão geral de morbidade e uso de serviços de saúde, as mulheres idosas rurais consultaram mais médicos $(76 \%$; IC: $74,2-77,7)$ do que os homens idosos residentes em áreas rurais (61,5\%; IC: 59,5-63,4). Entretanto, a proporção de pessoas que referiu consulta ao médico no último ano tendeu a aumentar com a idade entre os homens, mas não entre as mulheres (Tabela 2). Essas proporções foram bastante inferiores às observadas entre os idosos residentes em áreas urbanas: $84,7 \%$ (IC: $84,1-85,3$ ) nas mulheres e 73,5\% (IC: 72,6-74,4) nos homens (Tabela 3).

Assumiu-se que mais de uma consulta médica no ano indica continuidade do cuidado pela presença de consultas subseqüentes, que, principalmente entre os idosos, devem ocorrer em razão da alta prevalência de doenças crônicas. Em 2003, observou-se maior continuidade do 
Indicadores de morbidade referida entre pessoas com 60 anos e mais, residentes em áreas rurais e urbanas. Brasil, 2003.

\begin{tabular}{|c|c|c|c|c|c|c|c|c|}
\hline \multirow[t]{2}{*}{ Indicadores } & \multicolumn{2}{|c|}{ 60-69 anos } & \multicolumn{2}{|c|}{ 70-79 anos } & \multicolumn{2}{|c|}{80 anos e +} & \multicolumn{2}{|c|}{ Total } \\
\hline & $\%$ & IC95\% & $\%$ & IC95\% & $\%$ & IC95\% & $\%$ & IC95\% \\
\hline \multicolumn{9}{|l|}{ Rural } \\
\hline \multicolumn{9}{|c|}{ Prevalência de pessoas com auto-avaliação } \\
\hline \multicolumn{9}{|c|}{ ruim e muito ruim do estado de saúde } \\
\hline Homens & 11,1 & $9,5-13,0$ & 17,2 & $14,8-19,9$ & 27,1 & $22,9-31,7$ & 14,9 & $13,4-16,5$ \\
\hline Mulheres & 14,9 & $13,0-16,9$ & 20,6 & $17,7-23,8$ & 27,8 & $23,9-32,1$ & 18,4 & $16,7-20,2$ \\
\hline Total & 12,9 & $11,6-14,4$ & 18,8 & $16,8-21,1$ & 27,5 & $24,6-30,6$ & 16,6 & $15,3-18,0$ \\
\hline \multicolumn{9}{|c|}{$\begin{array}{l}\text { Prevalência de pessoas com restrição de } \\
\text { atividades rotineiras nos últimos } 15 \text { dias }\end{array}$} \\
\hline Homens & 9,7 & $8,2-11,4$ & 13,1 & $11,1-15,5$ & 20,2 & $16,2-24,9$ & 12,0 & $10,7-13,4$ \\
\hline Mulheres & 11,8 & $10,2-13,5$ & 12,8 & $10,5-15,5$ & 18,5 & $15,2-22,4$ & 13,0 & $11,7-14,4$ \\
\hline Total & 10,7 & $9,5-12,0$ & 13,0 & $11,3-14,8$ & 19,3 & $16,4-22,4$ & 12,5 & $11,4-13,6$ \\
\hline \multicolumn{9}{|c|}{ Prevalência de pessoas com incapacidade } \\
\hline \multicolumn{9}{|c|}{ para mobilidade física moderada } \\
\hline Homens & 9,6 & $8,3-11,0$ & 20,8 & $18,3-23,4$ & 44,0 & $38,4-49,7$ & 17,0 & $15,6-18,5$ \\
\hline Mulheres & 17,3 & $15,4-19,3$ & 29,2 & $25,9-32,8$ & 53,2 & $48,0-58,3$ & 25,9 & $24,0-27,8$ \\
\hline Total & 13,3 & $12,1-14,7$ & 24,9 & $22,5-27,4$ & 48,9 & $44,8-53,0$ & 21,4 & $20,0-22,8$ \\
\hline \multicolumn{9}{|l|}{ Urbano } \\
\hline \multicolumn{9}{|c|}{ Prevalência de pessoas com auto-avaliação } \\
\hline \multicolumn{9}{|c|}{ ruim e muito ruim do estado de saúde } \\
\hline Homens & 10,0 & $9,3-10,8$ & 14,8 & $13,5-16,1$ & 21,9 & $19,5-24,5$ & 12,8 & $12,1-13,5$ \\
\hline Mulheres & 10,8 & $10,1-11,6$ & 14,3 & $13,3-15,4$ & 18,8 & $17,1-20,7$ & 13,1 & $12,5-13,7$ \\
\hline Total & 10,5 & $9,9-11,1$ & 14,5 & $13,3-15,4$ & 20,0 & $18,5-21,6$ & 12,9 & $12,4-13,5$ \\
\hline \multicolumn{9}{|c|}{$\begin{array}{l}\text { Prevalência de pessoas com restrição de } \\
\text { atividades rotineiras nos últimos } 15 \text { dias }\end{array}$} \\
\hline Homens & 10,1 & $9,4-10,8$ & 13,4 & $12,3-14,6$ & 17,4 & $15,2-19,7$ & 11,9 & $11,3-12,6$ \\
\hline Mulheres & 12,6 & $11,8-13,4$ & 14,7 & $13,7-15,8$ & 20,2 & $18,4-22,1$ & 14,3 & $13,7-14,9$ \\
\hline Total & 11,5 & $10,9-12,0$ & 14,2 & $13,4-15,0$ & 19,1 & $17,7-20,7$ & 13,3 & $12,8-13,8$ \\
\hline \multirow{2}{*}{\multicolumn{9}{|c|}{$\begin{array}{l}\text { Prevalência de pessoas com incapacidade } \\
\text { para mobilidade física moderada }\end{array}$}} \\
\hline & & & & & & & & \\
\hline Homens & 11,3 & $10,5-12,1$ & 20,9 & $19,5-22,5$ & 43,2 & $40,2-46,3$ & 17,8 & $17,0-18,6$ \\
\hline Mulheres & 17,0 & $16,1-17,9$ & 30,5 & $29,0-32,0$ & 56,7 & $54,4-59,1$ & 26,7 & $25,9-27,6$ \\
\hline Total & 14,5 & $13,8-15,1$ & 26,5 & $25,3-27,7$ & 51,7 & $49,7-53,6$ & 22,9 & $22,2-23,6$ \\
\hline
\end{tabular}

Fonte: Instituto Brasileiro de Geografia e Estatística 12.

cuidado entre as mulheres rurais em comparação aos homens: $81 \%$ (IC: 79,0-82,8) e 74,5\% (IC: 72,4-76,5). A continuidade do cuidado aumentou com a idade em ambos os sexos (Tabela 2). Entretanto, a continuidade do cuidado foi maior nos idosos residentes em áreas urbanas para mulheres e homens respectivamente, $87,1 \%$ (IC: $86,4-$ 87,7 ) e $80,9 \%$ (IC: 79,9-81,8) (Tabela 3).

A cobertura de mamografia nos dois anos que antecederam a entrevista entre as mulheres de 60 a 69 anos representa um indicador de acesso e adequação da atenção primária neste grupo populacional. Essa cobertura limitou-se a 13,9\% (IC: 12,1-15,9) para as mulheres residentes em áreas rurais. Já em áreas urbanas, a cobertura por mamografia para mulheres nesse grupo etário foi de 43,1\% (IC: 41,9-44,3).

A proporção de idosos que referiu ter um serviço de saúde de uso regular, entre as pessoas residentes em áreas rurais, foi de 73,4\% (IC: 70,6$76,1)$ em 2003. Não se observou variação expressiva nessas proporções entre homens e mulheres e grupos etários (Tabela 2). Essa proporção foi também maior nos idosos residentes em áreas urbanas: 81,8\% (IC: 81,0-82,6) (Tabela 3).

A proporção de idosos rurais que consultou um dentista no último ano também foi muito baixa em 2003: 9,1\% (IC: 8,2-10,1). Essa proporção 
Indicadores de acesso e de utilização de serviços de saúde e fontes de financiamento do cuidado entre pessoas com 60 anos e mais, residentes em áreas rurais. Brasil, 2003

\begin{tabular}{|c|c|c|c|c|c|}
\hline Indicadores & Sexo & $\begin{array}{l}60-69 \text { anos } \\
\%(I C 95 \%)\end{array}$ & $\begin{array}{l}\text { 70-79 anos } \\
\%(I C 95 \%)\end{array}$ & $\begin{array}{c}80 \text { anos e + } \\
\%(I C 95 \%)\end{array}$ & $\begin{array}{c}\text { Total } \\
\% \text { (IC95\%) }\end{array}$ \\
\hline \multicolumn{6}{|l|}{ Acesso e uso de serviços } \\
\hline Proporção de pessoas que consultaram & Homens & $57,1(54,8-59,4)$ & $66,9(63,4-70,3)$ & $68,5(64,1-72,7)$ & $61,5(59,5-63,4)$ \\
\hline \multirow[t]{2}{*}{ médico nos últimos 12 meses } & Mulheres & $75,8(73,5-78,0)$ & $77,2(74,4-79,8)$ & $74,1(69,2-78,4)$ & $76,0(74,2-77,7)$ \\
\hline & Total & $66,2(64,4-67,9)$ & $71,9(69,4-74,3)$ & $71,5(68,1-74,7)$ & $68,6(67,0-70,2)$ \\
\hline Proporção de pessoas entre as que & Homens & $70,5(67,5-73,4)$ & $78,5(74,9-81,8)$ & $80,5(75,7-84,6)$ & $74,5(72,4-76,5)$ \\
\hline consultaram, que tiveram mais de uma & Mulheres & $80,1(77,6-82,5)$ & $80,5(77,0-83,6)$ & $85,6(81,0-89,2)$ & $81,0(79,0-82,8)$ \\
\hline consulta médica nos últimos 12 meses & Total & $75,9(73,9-77,7)$ & $79,6(77,2-81,8)$ & $83,3(80,2-86,0)$ & $78,0(76,6-79,4)$ \\
\hline Proporção de pessoas que referem & Homens & $79,4(75,0-83,3)$ & $83,3(76,9-88,2)$ & $74,0(63,3-82,5)$ & $79,7(75,9-83,1)$ \\
\hline \multirow[t]{2}{*}{ serviço de uso regular } & Mulheres & $82,4(75,7-87,6)$ & $80,2(74,7-84,8)$ & $78,9(70,6-85,3)$ & $81,1(76,9-84,6)$ \\
\hline & Total & $73,0(69,9-75,9)$ & $73,8(70,7-76,8)$ & $74,0(69,5-78,0)$ & $73,4(70,6-76,1)$ \\
\hline Proporção de pessoas que consultaram & Homens & $12,2(10,8-13,8)$ & $9,4(7,7-11,4)$ & $5,7(3,8-8,5)$ & $10,6(9,6-11,7)$ \\
\hline \multirow[t]{2}{*}{ dentista nos últimos 12 meses } & Mulheres & $8,9(7,4-10,7)$ & $7,0(5,3-9,0)$ & $3,6(2,1-6,2)$ & $7,6(6,4-8,9)$ \\
\hline & Total & $10,6(9,4-12,0)$ & $8,2(7,0-9,6)$ & $4,6(3,2-6,6)$ & $9,1(8,2-10,1)$ \\
\hline Taxa de utilização de serviços de saúde & Homens & $12,8(11,4-14,3)$ & $18,5(16,1-21,1)$ & $15,9(12,6-19,9)$ & $14,9(13,6-16,4)$ \\
\hline \multirow[t]{2}{*}{ nos últimos 15 dias por 100 pessoas } & Mulheres & $21,3(19,2-23,5)$ & $22,4(20,0-25,1)$ & $19,6(16,1-23,8)$ & $21,4(17,8-23,1)$ \\
\hline & Total & $16,9(15,6-18,3)$ & $20,4(18,6-22,4)$ & $17,9(15,2-21,0)$ & $18,1(16,9-19,4)$ \\
\hline Proporção de pessoas com restrição & Homens & $59,5(51,9-66,7)$ & $50,0(41,1-58,9)$ & $28,3(18,0-41,4)$ & $50,2(44,5-55,9)$ \\
\hline de atividades que usaram serviços & Mulheres & $63,6(56,0-70,5)$ & $53,2(43,9-62,2)$ & $42,4(32,4-53,1)$ & $56,3(51,0-61,5)$ \\
\hline nos últimos 15 dias & Total & $61,7(55,9-67,1)$ & $51,5(44,9-58,1)$ & $35,6(27,1-45,0)$ & $53,3(49,0-57,6)$ \\
\hline Taxa de internação hospitalar nos & Homens & $9,9(8,7-11,2)$ & $16,0(13,8-18,5)$ & $16,9(13,2-21,5)$ & $12,6(11,4-13,9)$ \\
\hline \multirow[t]{2}{*}{ últimos 12 meses } & Mulheres & $12,4(10,8-14,2)$ & $15,1(12,6-18,0)$ & $15,5(12,6-19,1)$ & $13,6(12,2-15,3)$ \\
\hline & Total & $11,1(10,0-12,2)$ & $15,6(13,8-17,5)$ & $16,2(13,7-19,0)$ & $13,1(12,0-14,2)$ \\
\hline Proporção de pessoas com & Homens & $9,0(7,7-10,4)$ & $8,7(6,9-10,9)$ & $8,0(5,6-10,9)$ & $8,8(7,8-9,9)$ \\
\hline \multirow[t]{2}{*}{ plano de saúde } & Mulheres & $9,0(7,5-10,7)$ & $1,3(9,0-14,1)$ & $6,7(4,6-9,2)$ & $9,3(8,0-10,9)$ \\
\hline & Total & $9,0(7,8-10,3)$ & $10,0(8,2-12,0)$ & $7,1(5,3-9,6)$ & $9,1(8,0-10,2)$ \\
\hline \multicolumn{6}{|l|}{ Fonte de financiamento do cuidado } \\
\hline Plano de saúde & & $8,3(6,3-10,9)$ & $7,8(5,0-11,9)$ & $8,4(4,8-14,3)$ & $8,2(6,4-10,4)$ \\
\hline SUS & & $70,6(66,9-74,1)$ & $65,7(60,1-70,9)$ & $77,8(66,9-84,8)$ & $69,8(66,5-72,9)$ \\
\hline Próprio bolso & & $14,0(11,2-17,4)$ & $21,9(17,7-26,7)$ & $14,5(8,4-23,9)$ & $16,8(14,3-19,6)$ \\
\hline
\end{tabular}

Fonte: Instituto Brasileiro de Geografia e Estatística 12

diminuiu linearmente com a idade, em ambos os gêneros. Entretanto, contrariamente ao observado nas consultas médicas, os homens rurais consultaram mais o dentista do que as mulheres rurais, em todos os grupos de idade (Tabela 2). Embora muito baixa, dentre idosos residentes nas áreas urbanas, essa proporção é mais do que o dobro da observada nas áreas rurais: $19,1 \%$ (IC: 18,4-19,8). As diferenças de gênero também são menores nas áreas urbanas: 20,1\% (IC: 19,3-21,0) nos homens e 18,3\% (IC: 17,5-19,1) nas mulheres (Tabela 3).
Em 2003, os postos e centros de saúde predominaram dentre os serviços procurados pelos idosos residentes em áreas rurais $(48,1 \%$; IC: $44,1-52,2)$, seguidos dos hospitais $(25,2 \%$; IC: $21,6-29,1)$ e consultórios privados $(12,7 \%$; IC: $10,3-15,4)$. A farmácia (3,5\%; IC: $2,4-5,0)$ e o atendimento domiciliar (2,8\%; IC: 1,7-4,4) apresentaram participação relativamente baixa. Nos idosos residentes em áreas urbanas, apesar de predominar a participação dos postos e centros de saúde (36,5\%; IC: 35,1-38,0), o peso relativo dos consultórios privados é maior (24\%; IC: 22,7 - 
Indicadores de acesso e de utilização de serviços de saúde e fonte de financiamento entre pessoas com 60 anos e mais, residentes em áreas urbanas. Brasil 2003.

\begin{tabular}{|c|c|c|c|c|c|}
\hline Indicadores & Sexo & $\begin{array}{l}\text { 60-69 anos } \\
\%(I C 95 \%)\end{array}$ & $\begin{array}{l}\text { 70-79 anos } \\
\% \text { (IC95\%) }\end{array}$ & $\begin{array}{c}80 \text { anos e + } \\
\%(I C 95 \%)\end{array}$ & $\begin{array}{c}\text { Total } \\
\% \text { (IC95\%) }\end{array}$ \\
\hline \multicolumn{6}{|l|}{ Acesso e uso de serviços } \\
\hline Proporção de pessoas que consultaram & Homens & $70,4(69,1-71,6)$ & $76,8(75,4-78,2)$ & $80,4(78,0-82,5)$ & $73,5(72,6-74,4)$ \\
\hline \multirow[t]{2}{*}{ médico nos últimos 12 meses } & Mulheres & $84,2(83,3-85,0)$ & $85,4(84,3-86,4)$ & $85,1(83,5-86,6)$ & $84,7(84,1-85,3)$ \\
\hline & Total & $78,1(77,3-78,8)$ & $81,8(80,9-82,6)$ & $83,3(82,0-84,6)$ & $79,9(79,3-80,5)$ \\
\hline Proporção de pessoas entre as que & Homens & $78,7(77,3-80,0)$ & $83,7(82,3-85,1)$ & $83,3(80,7-85,6)$ & $80,9(79,9-81,8)$ \\
\hline consultaram, que tiveram mais de uma & Mulheres & $86,4(85,4-87,2)$ & $88,1(87,0-89,1)$ & $87,5(85,8-89,1)$ & $87,1(86,4-87,7)$ \\
\hline consulta médica nos últimos 12 meses & Total & $83,3(82,5-84,1)$ & $86,4(85,5-86,7)$ & $86,0(84,5-87,3)$ & $84,7(84,0-85,2)$ \\
\hline Proporção de pessoas que referem & Homens & $79,0(77,7-80,1)$ & $79,6(78,0-81,0)$ & $79,8(77,2-82,1)$ & $79,2(78,2-80,2)$ \\
\hline \multirow[t]{2}{*}{ serviço de uso regular } & Mulheres & $83,5(82,5-84,5)$ & $84,0(82,8-85,1)$ & $83,9(82,1-85,6)$ & $83,7(82,9-84,5)$ \\
\hline & Total & $81,5(80,6-82,4)$ & $82,1(81,0-83,2)$ & $82,4(80,8-83,9)$ & $81,8(81,0-82,6)$ \\
\hline Proporção de pessoas que consultaram & Homens & $23,5(22,3-24,7)$ & $17,3(15,9-18,7)$ & $10,7(9,0-12,7)$ & $20,1(19,3-21,0)$ \\
\hline \multirow[t]{2}{*}{ dentista nos últimos 12 meses } & Mulheres & $21,9(20,8-23,0)$ & $16,1(14,9-17,3)$ & $9,3(8,0-10,8)$ & $18,3(17,5-19,1)$ \\
\hline & Total & $22,6(21,7-23,5)$ & $16,6(15,6-17,6)$ & $9,9(8,7-11,1)$ & $19,1(18,4-19,8)$ \\
\hline Taxa de utilização de serviços de saúde & Homens & $19,7(18,7-20,8)$ & $24,6(23,1-26,2)$ & $26,0(23,5-28,8)$ & $22,0(21,1-22,8)$ \\
\hline \multirow[t]{2}{*}{ nos últimos 15 dias por 100 pessoas } & Mulheres & $27,3(26,3-28,3)$ & $28,9(27,6-30,3)$ & $26,8(24,8-28,9)$ & $27,8(27,0-28,5)$ \\
\hline & Total & $23,9(23,2-24,7)$ & $27,1(26,1-28,2)$ & $26,5(24,9-28,2)$ & $25,3(24,7-25,9)$ \\
\hline Proporção de pessoas com restrição & Homens & $59,8(55,9-63,6)$ & $60,1(55,6-64,4)$ & $57,8(50,6-64,7)$ & $59,6(56,9-62,2)$ \\
\hline de atividade que usaram serviços nos & Mulheres & $63,9(60,9-66,9)$ & $63,4(59,7-67,0)$ & $55,9(50,9-60,4)$ & $62,3(60,0-64,5)$ \\
\hline últimos 15 dias & Total & $62,3(59,9-64,7)$ & $62,1(59,2-64,9)$ & $56,6(52,5-60,5)$ & $61,2(59,4-63,0)$ \\
\hline Taxa de internação hospitalar nos & Homens & $10,7(10,0-11,5)$ & $15,4(14,3-16,7)$ & $20,2(18,2-22,7)$ & $13,2(12,6-13,9)$ \\
\hline \multirow[t]{2}{*}{ últimos 12 meses } & Mulheres & $10,0(9,3-10,7)$ & $13,6(12,6-14,7)$ & $17,8(16,1-19,7)$ & $12,2(11,6-12,8)$ \\
\hline & Total & $10,3(9,7-10,9)$ & $14,4(13,6-15,2)$ & $18,7(17,3-20,2)$ & $12,6(12,2-13,1)$ \\
\hline Proporção de pessoas com plano & Homens & $31,2(29,8-32,5)$ & $33,4(31,6-35,3)$ & $29,1(26,5-32,0)$ & $31,7(30,5-32,8)$ \\
\hline \multirow[t]{2}{*}{ de saúde } & Mulheres & $34,6(33,3-36,0)$ & $35,8(34,1-37,4)$ & $34,1(31,7-36,6)$ & $34,9(33,8-36,1)$ \\
\hline & Total & $33,1(32,0-34,2)$ & $34,8(33,4-36,3)$ & $32,2(30,0-34,2)$ & $33,5(32,5-34,6)$ \\
\hline \multicolumn{6}{|c|}{ Fonte de financiamento dos atendimentos ambulatoriais } \\
\hline Plano de saúde & & $29,2(27,5-31,0)$ & $32,0(29,7-34,3)$ & $28,1(24,8-31,2)$ & $30,0(28,6-31,5)$ \\
\hline SUS & & $55,4(53,5-57,3)$ & $51,1(48,7-53,4)$ & $53,6(49,9-57,2)$ & $53,7(52,1-55,2)$ \\
\hline Próprio bolso & & $12,9(11,8-14,1)$ & $14,9(13,3-16,5)$ & $15,6(13,1-18,5)$ & $13,9(13,0-14,9)$ \\
\hline
\end{tabular}

Fonte: Instituto Brasileiro de Geografia e Estatística 12.

$25,4)$ e dos hospitais, menor (19,6\%; IC: 18,5 $20,8)$. O mesmo pode ser dito da farmácia $(1,2 \%$; IC: $0,9-1,5)$ e do atendimento domiciliar $(1,7 \%$; IC: 1,4-2,1) (Figura 1).

O padrão de acesso reflete-se na utilização de serviços de saúde. A proporção de idosos rurais que utilizou serviços de saúde nos quinze dias que antecederam a entrevista foi de18,1\% (IC: 16,9-19,4) em 2003 (Tabela 2). No caso dos idosos urbanos, a taxa de utilização de serviços também foi bem maior: 25,3 por 100 idosos (IC: 24,7-25,9) (Tabela 3).
Na presença de necessidade de saúde, inferida pela resposta positiva à variável "restrição de atividade por motivo de saúde nos 15 dias que antecederam a entrevista", a taxa de utilização de serviços de saúde aumenta. A taxa de utilização nos 15 dias que antecederam a entrevista entre os idosos rurais que referiram necessidade foi 53,3 por 100 idosos (IC: 49,0-57,6) e foi menor entre os mais idosos, de 80 anos e mais: 35,6 por 100 idosos. As idosas rurais com restrição de atividade utilizaram mais os serviços de saúde do que os homens, 56,3 por 100 idosas (IC: $51,0-61,5$ ) e 50,2 
Figura 1

Tipos de serviços procurados para atendimento de saúde nos últimos 15 dias, por pessoas com 60 anos e mais. Brasil, 2003.

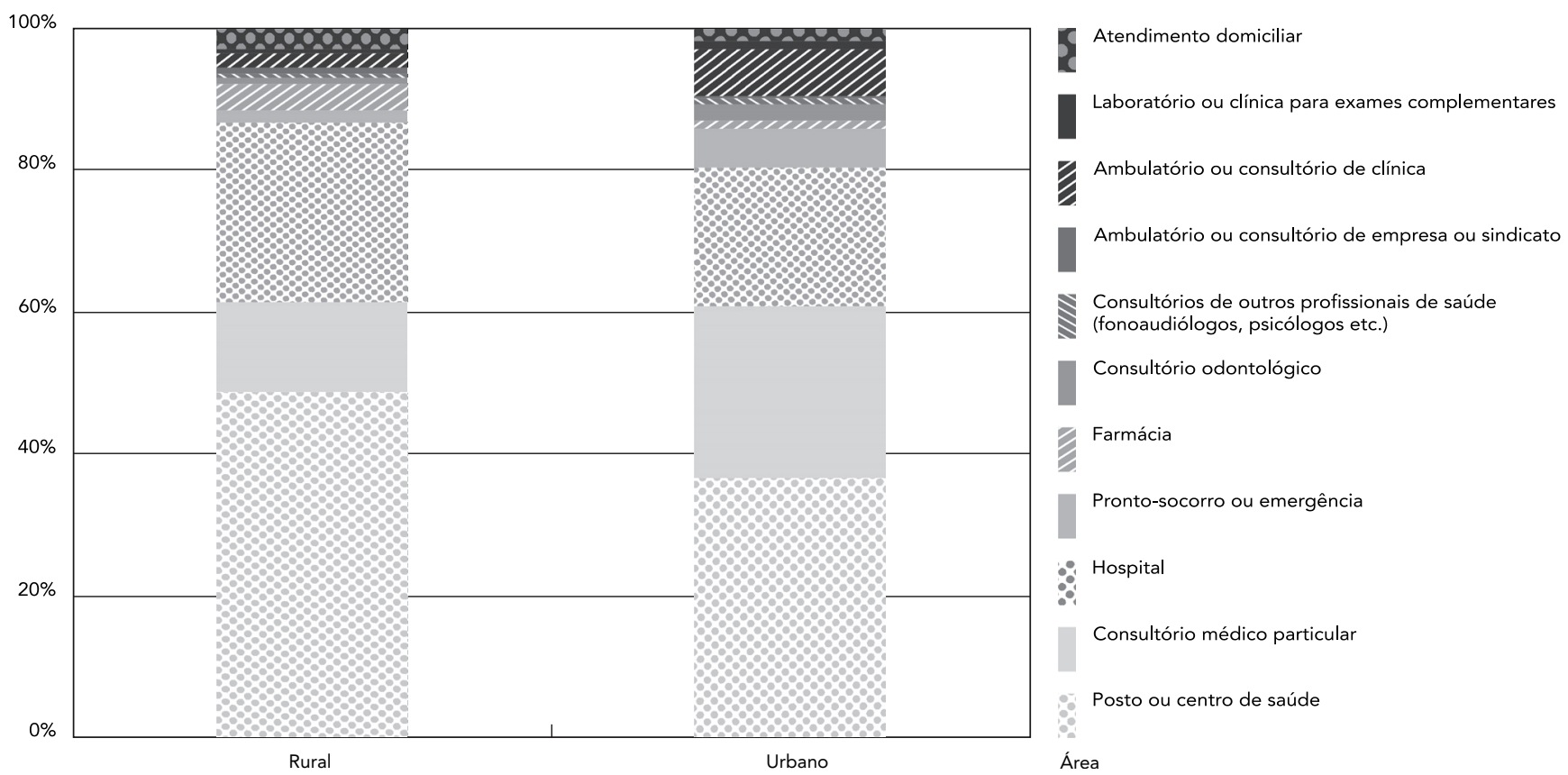

(IC: 44,5-55,9), respectivamente. Esse diferencial de gênero deve-se à variação no uso de serviços de saúde entre mulheres e homens com 80 anos e mais. Os idosos urbanos com restrição de atividades apresentaram maior taxa de utilização de serviço do que os idosos rurais: 61,2 por 100 idosos (IC: 59,4-63,0). Os idosos urbanos com 80 anos e mais também utilizaram menos serviços de saúde (56,6 por 100 idosos) do que os idosos entre 60 e 79 anos (Tabelas 2 e 3).

Os dados da PNAD não indicam presença de demanda reprimida, já que o percentual de pessoas idosas que procuraram serviços e não foram atendidas foi menor do que $1 \%$, tanto nas áreas rurais como nas urbanas. Dessa forma, o diferencial na utilização resulta de variação na procura por serviços de saúde, com os idosos residentes em áreas rurais procurando menos do que os urbanos.

A taxa de internação no último ano entre os idosos rurais foi de 13,1 por 100 idosos (IC: 12,0$14,2)$ em 2003. Variou pouco entre homens e mulheres, mas aumentou linearmente com a idade: 11,1 por 100 (IC: 10,0-12,2) nos idosos entre $60 \mathrm{e}$ 69 anos de idade; 15,6 por 100 (IC: $13,8-17,5$ ) nos idosos entre 70 e 79 anos de idade; e 16,2 por 100
(IC: 13,7-19,0) nos idosos com 80 anos e mais. Nas áreas urbanas, a taxa geral de internação não diferiu da área rural (12,6\%; IC: 12,2-13,1), mas os homens idosos residentes nas áreas urbanas apresentaram taxas de internação maiores do que as das mulheres, em particular nos grupos de idade mais avançada (Tabelas 2 e 3 ).

Em 2003, o SUS financiou a maior parte dos atendimentos realizados nos 15 dias que antecederam a entrevista, tendo pagado $69,8 \%$ (IC: 66,5-72,9) do total de atendimentos dos idosos residentes em áreas rurais. No entanto, parcela expressiva desses idosos rurais (16,8\%; IC: 14,319,6) referiu ter pagado do próprio bolso, em parte ou integralmente, pelo serviço recebido. O SUS pagou uma menor proporção (53,7\%: IC: 52,1-55,2) dos atendimentos aos idosos urbanos no mesmo ano. No entanto, uma proporção menor de idosos urbanos pagou do próprio bolso pelos atendimentos recebidos: 13,9\% (IC: 13,014,9). Diferenças no tipo de financiamento associaram-se à variação na cobertura de plano de saúde: 9,1\% (IC: 8,0-10,2) entre os idosos rurais e $33,5 \%$ (IC: $32,5-34,6$ ) entre os idosos urbanos. Os planos de saúde pagaram 8,2\% (IC: 6,4-10,4) dos atendimentos aos idosos residentes em áreas 
rurais e $30 \%$ (IC: $28,6-31,5)$ aos idosos residentes em áreas urbanas.

Em relação ao desempenho dos serviços de saúde na atenção à população rural no período compreendido entre 1998 e 2003, observou-se que houve alguma melhora (Tabelas 2 e 4). A proporção de idosos que referiu ter consultado um médico no último ano aumentou. $\mathrm{O}$ mesmo aconteceu com a continuidade do cuidado, que aumentou em ambos os sexos. $\mathrm{O}$ aumento na proporção de idosos que consultou médico no último ano foi acompanhado do aumento da proporção de idosos que referiu ter um serviço de saúde de uso regular. Essas variações ocorreram em ambos os gêneros e em todas as idades. $\mathrm{O}$ aumento de serviços de uso regular ocorreu, em particular, nos postos e centros de saúde. A proporção de idosos que consultou um dentista no último ano, apesar de continuar muito baixa, também apresentou leve aumento entre as duas pesquisas. O mesmo foi observado na taxa de utilização de serviços de saúde nos quinze dias que antecederam a entrevista, entre os idosos residentes em áreas rurais que referiram ou não restrição de atividade por motivo de saúde. O SUS aumentou sua participação enquanto fonte de pagamento dos serviços utilizados, paralelamente à redução do pagamento do próprio bolso. Houve aumento expressivo da participação relativa dos postos e centros de saúde na prestação de serviços, mas não na redução da participação dos hospitais. A cobertura por plano de saúde também aumentou, mas não a sua participação como fonte de pagamento. O coeficiente de internação de idosos rurais não apresentou variação significativa no período.

\section{Discussão}

Este estudo voltou-se para avaliar o padrão de acesso e utilização de serviços de saúde dos idosos residentes em áreas rurais no Brasil no ano de 2003. Em consonância com o padrão observado

\begin{tabular}{|c|c|c|c|}
\hline Indicadores & $\begin{array}{l}\text { Homens } \\
\%(I C 95 \%)\end{array}$ & $\begin{array}{l}\text { Mulheres } \\
\%(I C 95 \%)\end{array}$ & $\begin{array}{c}\text { Total } \\
\%(\text { IC95\%) }\end{array}$ \\
\hline \multicolumn{4}{|l|}{ Acesso e uso de serviços } \\
\hline $\begin{array}{l}\text { Proporção de pessoas que fizeram consulta } \\
\text { médica nos últimos } 12 \text { meses }\end{array}$ & $55,9(53,4-58,2)$ & $69,9(67,3-72,3)$ & $62,8(60,7-64,8)$ \\
\hline $\begin{array}{l}\text { Proporção de pessoas entre as que consultaram, } \\
\text { que tiveram mais de uma consulta médica } \\
\text { nos últimos } 12 \text { meses }\end{array}$ & $70,5(67,8-73,1)$ & $77,5(75,3-79,6)$ & $74,4(72,5-76,2)$ \\
\hline $\begin{array}{l}\text { Proporção de pessoas que utilizam } \\
\text { normalmente o mesmo serviço }\end{array}$ & $64,0(60,2-67,6)$ & $68,3(64,4-71,9)$ & $66,1(62,5-69,6)$ \\
\hline $\begin{array}{l}\text { Proporção de pessoas que consultaram dentista } \\
\text { nos últimos } 12 \text { meses }\end{array}$ & $8,5(7,2-9,9)$ & $4,9(4,1-5,8)$ & $6,7(5,8-7,7)$ \\
\hline Proporção de pessoas que usaram serviços de saúde & $12,2(10,9-13,7)$ & $16,6(14,9-18,4)$ & $14,4(13,1-15,7)$ \\
\hline $\begin{array}{l}\text { Proporção de pessoas com restrição de atividade } \\
\text { rotineira que usaram serviços de saúde }\end{array}$ & $42,9(37,8-48,3)$ & $43,6(38,3-49,0)$ & $43,3(39,3-47,3)$ \\
\hline Taxa de internação hospitalar nos últimos 12 meses & $12,2(10,9-13,6)$ & $13,6(12,2-15,2)$ & $12,9(11,8-14,1)$ \\
\hline Cobertura por plano de saúde & $5,8(4,7-7,2)$ & $6,3(5,1-7,9)$ & $6,1(4,9-7,5)$ \\
\hline Fonte de financiamento do cuidado & & & \\
\hline Plano de saúde & $5,6(3,4-8,9)$ & $7,3(6,3-11,7)$ & $7,3(5,7-9,3)$ \\
\hline SUS & $59,6(54,1-64,8)$ & $57,8(53,1-62,4)$ & $58,6(55,0-62,0)$ \\
\hline Próprio bolso & $21,1(17,0-25,7)$ & $19,5(16,0-23,5)$ & $20,2(17,5-23,1)$ \\
\hline
\end{tabular}

Fonte: Instituto Brasileiro de Geografia e Estatística ${ }^{3}$. 
em populações rurais, descrito por outras pesquisas 7,8 , verificou-se que a população de idosos rurais no Brasil também se encontrava em situação de marcada desvantagem no acesso e na utilização de serviços de saúde em comparação à população de idosos urbanos.

A proporção de idosos que consultou o médico no último ano, a continuidade do cuidado, a proporção que consultou o dentista e a cobertura de mamografia observadas nos idosos rurais foram expressivamente mais baixas do que nos idosos urbanos. Mesmo analisando aqueles que referiram problema de saúde, a utilização de serviços de saúde foi menor nos idosos rurais do que nos urbanos; mas não se observou diferença nas taxas de internação entre esses dois grupos.

Cerca de três quartos dos idosos residentes em áreas rurais tinham um serviço de saúde que utilizavam regularmente. Os principais serviços de saúde utilizados regularmente para atendimento ambulatorial eram os Postos e Centros de Saúde e os Hospitais. Esse padrão possivelmente reflete a oferta de serviços disponível a essa população à época do estudo, polarizada entre serviços de baixa complexidade e o hospital, o que reflete a carência de serviços especializados. Essa conformação da oferta de serviços resulta em reduzida eficiência e baixa adequação dos cuidados prestados. Acesso limitado a serviços de complexidade intermediária no SUS e as barreiras financeiras no acesso a médicos especialistas em consultórios privados resultam em agravamento desnecessário do estado de saúde dos idosos e maior demanda aos hospitais. Esse agravamento resultaria de cuidados inadequados em serviços de baixa complexidade ou da postergação da procura por parte da população ao perceber a inadequação às suas necessidades dos serviços a elas disponíveis. Dados de diversos países 19 mostram que o acesso a cuidados especializados é mais iníquo do que o acesso a médicos generalistas. Essa iniqüidade é particularmente danosa aos idosos que, por sua maior fragilidade biológica, requerem cuidados especializados em maior intensidade.

Os idosos com 80 anos ou mais residentes em áreas rurais aparentemente enfrentam barreiras de acesso aos serviços de saúde ainda maiores, pois utilizam menos os serviços de saúde quando necessitam do que os idosos de menor idade. Esse é o grupo etário que apresenta piores condições de saúde. Cerca de $50 \%$ referem incapacidade para mobilidade física moderada. Fatores associados à distância aos serviços e às dificuldades de deslocamento restringem ainda mais o acesso dos mais idosos. No caso dos idosos residentes em áreas rurais, essas restrições tornamse mais acentuadas dada a dispersão territorial das pessoas e a dificuldade de localizar serviços próximos às suas residências. Fatores culturais também são importantes para que os serviços sejam permeáveis aos valores e às preferências da população de idosos rurais. A melhoria do acesso, nesse caso, implica arranjos organizacionais do sistema local de saúde de modo a adaptar os serviços às necessidades e incapacidades desse grupo etário. A distância dos serviços pode ser compensada por medidas como a facilitação de transporte, a presença de sistema de referência aos serviços hospitalares e mecanismos de acolhimento que facilitem a obtenção de cuidado. Estudo realizado no Estado de Nova Iorque, Estados Unidos, após implantação de mudanças voltadas para melhorar o acesso aos hospitais da população rural, sugere que estas foram capazes de diminuir o efeito da distância na propensão de procura de cuidado à saúde de residentes em áreas rurais com condições de saúde graves 20 .

No caso dos idosos rurais no Brasil, verificouse que as taxas de utilização de serviços de saúde não eram compatíveis com suas necessidades de saúde, nem o cuidado era de boa qualidade. A taxa de utilização nos quinze dias que antecederam a entrevista dos idosos que referiram problema de saúde no período foi de 53,3 por 100 idosos rurais e foi de 61,2 por 100 idosos urbanos. Entre as mulheres idosas residentes nas áreas rurais com 60 a 69 anos, 75,8\% tiveram pelo menos uma consulta médica no último ano; dessas, $80,1 \%$ realizaram mais de uma consulta no mesmo período. Mesmo assim, apenas 13,9\% referiram ter realizado uma mamografia nos dois anos que antecederam a entrevista.

A cobertura de mamografia nas mulheres idosas foi também baixa na população urbana, porém mais alta que na população rural. Esse fato torna-se relevante na medida em que os profissionais de saúde podem estar sistematicamente excluindo os idosos de cuidados recomendados, cujos benefícios seriam por eles minimizados em função da avançada idade dos pacientes e da alta prevalência de co-morbidades. Há na literatura evidência de que os idosos recebem cuidados de qualidade inferior aos prestados a pessoas mais jovens. Em particular, em locais com marcada limitação de recursos, como ocorre com mais intensidade nas áreas rurais, a seleção de pacientes na indicação de procedimentos de maior complexidade tende a desfavorecer grupos socialmente mais vulneráveis 21 .

O nível educacional do paciente é um fator importante no acesso e na adequação do cuidado prestado. As pessoas de maior escolaridade detêm mais informação e mais recursos de comunicação, aos quais recorrem na sua relação com os serviços e profissionais de saúde. Os ido- 
sos rurais têm baixa escolaridade e são, assim, menos capacitados para identificar os serviços de que necessitam e mais vulneráveis à discriminação por parte dos profissionais de saúde. Barreiras de informação 22 devem ser particularmente críticas nesse caso e certamente devem ser objeto de políticas voltadas para melhorar o acesso aos cuidados preventivos e curativos da população idosa. A 2a Assembléia Mundial sobre o envelhecimento destacou a necessidade de se acabar com a discriminação em função da idade para reduzir barreiras de acesso aos serviços de saúde enfrentadas pelos idosos 1 .

Em consonância com o padrão na população em geral 4, as mulheres idosas residentes em áreas rurais auto-avaliaram seu estado de saúde pior do que os homens e, na maioria dos casos, utilizaram mais serviços de saúde. Mesmo quando ajustada por necessidade, a taxa de utilização das mulheres permaneceu significativamente maior do que a dos homens. A continuidade do cuidado também foi mais alta entre idosas rurais do que entre os idosos rurais, o que pode significar maior propensão das mulheres de procurar cuidado de saúde, mas também indicar maior inclinação dos médicos a recomendar retorno às mulheres. No caso da continuidade do cuidado, o papel da decisão médica é fundamental. Não houve variação de gênero na internação hospitalar, com exceção do grupo entre 60 e 69 anos em que as idosas apresentam maiores taxas.

O diferencial de gênero na utilização de serviços de saúde observado nos idosos residentes em áreas rurais seguiu aquele observado entre idosos residentes nas áreas urbanas, mas é menos marcado nestas. Dentre os idosos urbanos, observou-se em alguns indicadores diferencial de gênero na utilização de serviços que tendeu a favorecer os homens. Há divergência na literatura sobre os fatores que explicam as variações de gênero no padrão de utilização de serviços de saúde, e alguns autores propõem que estes sofrem influência do contexto social e cultural 23. O menor diferencial de gênero na utilização de serviços de saúde nos idosos urbanos fala em favor da tese que fatores sociais e culturais estariam na base das diferenças na percepção de morbidade e na decisão de procurar serviços de saúde dos idosos rurais, fatores estes que se alterariam com a urbanização.

Entre os idosos rurais com 80 ou mais anos de idade o diferencial de gênero na auto-avaliação do estado de saúde desapareceu. Não houve variação entre mulheres e homens idosos na referência à restrição de atividades por motivo de saúde, e as variações de gênero na utilização não foram expressivas. Os idosos com maior idade e aqueles que se internam estão entre os mais frágeis biologicamente. Nas pessoas com mais de 65 anos, em particular, a percepção da gravidade do problema de saúde relaciona-se a maiores taxas de utilização de serviços de saúde 24. Maiores taxas de internação hospitalar nos homens idosos já foram descritas em outros contextos e podem indicar diferença no perfil de morbidade de homens e mulheres idosas, com os homens idosos apresentando problemas de saúde mais graves 25,26 , mas podem também refletir a ocorrência de seleção de gênero na decisão médica, com os médicos sendo mais propensos a internar homens idosos do que mulheres idosas 23 .

Na utilização de dentista também houve inversão do padrão de gênero. A proporção de idosos que consultou o dentista no último ano foi maior que a proporção de idosas. Esse diferencial era mais acentuado em 1998 e, apesar de mais atenuado, estava presente também dentre os idosos residentes nas áreas urbanas em 2003. Esteve também presente em todas as faixas de idade analisadas neste trabalho. Estudo baseado em dados da PNAD 19984 mostra que nos residentes nas áreas urbanas a proporção de consulta ao dentista no último ano era favorável às mulheres nos idosos até os 64 anos, invertendo-se a partir dos 65 anos de idade, com a proporção de homens que consultou o dentista ultrapassando a proporção de mulheres. No caso dos residentes nas áreas rurais, a inversão no padrão de gênero ocorria em idade mais jovem, a partir dos 50 anos de idade. Concomitantemente, os homens, em praticamente todas as faixas de idade, apresentavam as maiores proporções de pessoas que nunca consultaram o dentista. Resumindo, os homens idosos consultavam mais o dentista que as mulheres idosas, mas uma grande proporção deles - maior do que entre as mulheres - nunca havia ido ao dentista. Possivelmente, o menor uso ao longo da vida dentre os homens explicaria a presença de problemas odontológicos mais graves na velhice que ocasionariam maior uso. Em estudo que empregou dados da PNAD 1998, Matos et al. 27 não observaram efeito independente de gênero entre os idosos na ida ao dentista no último ano, em modelo que incluiu variáveis geográficas, sociais e tipo de respondente - próprio ou substituto.

Em 2003, o SUS tinha expressiva participação no financiamento do cuidado de saúde prestado à população idosa rural e os planos de saúde mostravam pequena participação, reflexo da baixa cobertura de planos observada nesta população. A cobertura de plano privado de saúde era ainda mais baixa entre os idosos com mais de 79 anos. Apesar da participação do SUS, o paga- 
mento de cuidados de saúde com recursos próprios era alto entre os idosos residentes nas áreas rurais, o que denota a importância das barreiras financeiras no acesso aos serviços de saúde, que se mostraram ainda maiores do que as experimentadas pelos idosos urbanos.

A redução das barreiras financeiras com a expansão da rede de serviços do SUS e a redução de barreiras organizacionais com adequação desta rede às necessidades e às características de população idosa rural, fazem-se imperiosas para alterar as limitações de acesso aos serviços de saúde experimentadas por esta população. A predominância de pessoas de baixo poder aquisitivo nessa população e a ausência de vínculo empregatício são fatores estruturais que limitam o acesso aos serviços privados sem financiamento do SUS.

A melhora no desempenho dos serviços de saúde à população de idosos rurais em geral seguiu o mesmo padrão descrito para a população brasileira 12. Essa melhora deveu-se ao aumento da utilização de serviços de saúde, em particular pelo uso de serviços prestados em Postos e Centros de Saúde, com aumento na participação do SUS no financiamento dos serviços. Houve alguma redução nas barreiras financeiras indicada pela menor participação dos recursos do próprio bolso no pagamento de serviços de saúde. Ressalta-se um pequeno aumento na cobertura de plano de saúde, que não foi observado para a população em geral ${ }^{8}$. No entanto, contrariamente ao esperado, o aumento na cobertura de planos de saúde na população de idosos rurais não teve reflexo no aumento da participação dos planos de saúde privados no financiamento dos serviços prestados. Esse achado coloca em questão a qualidade dos planos de saúde privados disponíveis para essa população.
Em estudos que focam a população rural, é importante ressaltar que, no Brasil, a delimitação do perímetro das áreas urbanas é atribuição dos municípios, o que implica a possibilidade de grande variação nos critérios utilizados entre municípios. Assim, é possível encontrar áreas legalmente definidas como urbanas que ainda não foram de fato urbanizadas, e outras que, embora ainda definidas como rurais, já foram urbanizadas. Cabe lembrar que a classificação urbana e rural empregada neste artigo buscou discriminar melhor essas populações e difere da adotada pelo IBGE. Incluíram-se os residentes de área não urbanizada de cidade ou vila à população rural e excluíram-se da população urbana os residentes em aglomerados rurais de extensão urbana.

Os dados da PNAD apresentados neste artigo mostram que as populações territorialmente definidas como rural e urbana são marcadamente diferentes no que tange ao padrão de acesso e utilização de serviços de saúde, o que denota a importância do território nesse padrão. Como a amostra da PNAD exclui a população rural da Região Norte, que certamente experimenta maiores barreiras de acesso aos serviços de saúde por viver em áreas caracterizadas por grandes distâncias, dificuldades de transporte e grande concentração de pobreza, sugere-se que essas diferenças observadas sejam ainda mais marcadas.

O estudo mostrou que as políticas de saúde no período foram abrangentes, pois englobaram as populações de idosos rurais, ainda que limitadas para alterar a situação de desvantagem no acesso e na utilização de serviços de saúde desta população. A redução dessas desvantagens implica melhorar a oferta de serviços para a população rural, além de organizá-los de modo a atender às especificidades territoriais, culturais e sociais desses grupos populacionais. 


\section{Resumo}

Analisou-se o acesso aos serviços de saúde e sua utilização por idosos residentes em áreas rurais no Brasil em 2003, comparando os padrões observados com idosos residentes em área urbana e com o padrão existente em 1998, a partir dos dados dos suplementos sobre Acesso e Utilização de Serviços de Saúde da Pesquisa Nacional por Amostra de Domicílios. Observou-se que as barreiras de acesso eram maiores na área rural em comparação à urbana. A utilização de serviços de saúde era menor do que nos idosos urbanos, mesmo para aqueles que referiram problema de saúde. Não se observou diferença nas taxas de internação entre idosos rurais e urbanos. A análise do tipo de serviço utilizado mostrou que há acesso limitado a serviços de complexidade intermediária. Os resultados sugerem que os mais idosos apresentam barreiras de acesso ainda maiores. O diferencial de gênero na utilização favorável à mulher foi mais marcado nos idosos rurais. Barreiras financeiras também são mais marcadas. Houve indicativo de alguma melhora no desempenho dos serviços do Sistema Único de Saúde (SUS). Recomendou-se ampliação da oferta e adaptação dos serviços às especificidades territoriais, culturais e sociais dos idosos rurais.

Idoso; Acesso aos Serviços de Saúde; Pesquisa sobre Serviços de Saúde; Saúde da População Rural

\section{Referências}

1. Hoskins I, Kalache A, Mende S. Hacia una atención primaria de salud adaptada a las personas de edad. Rev Panam Salud Pública 2005; 17:444-51.

2. Donabedian A. An introduction to quality assurance in health care. New York: Oxford University Press; 2003.

3. Instituto Brasileiro de Geografia e Estatística. Acesso e utilização de serviços de saúde - PNAD, 1998. Rio de Janeiro: Instituto Brasileiro de Geografia e Estatística; 2000.

4. Pinheiro RS, Viacava F, Travassos C, Brito AS. Gênero, morbidade, acesso e utilização de serviços de saúde no Brasil. Ciênc Saúde Coletiva 2002; 7:687-707.

5. Lima-Costa MF, Barreto SM, Giatti L. Condições de saúde, capacidade funcional, uso de serviços de saúde e gastos com medicamentos da população idosa brasileira: um estudo descritivo baseado na Pesquisa Nacional por Amostra de Domicílios. Cad Saúde Pública 2003; 19:735-43.

\section{Colaboradores}

Ambos os autores realizaram em conjunto a concepção, a análise dos dados, a discussão e a redação do artigo.
6. Veras R. Em busca de uma assistência adequada à saúde do idoso: revisão da literatura e aplicação de um instrumento de detecção precoce e de previsibilidade de agravos. Cad Saúde Pública 2003; 19:705-15.

7. Rosenthal T, Fox C. Access to health care for the rural elderly. JAMA 2000; 284:2034-6.

8. Lishner DM, Rosenblatt RA, Baldwin L, Hart LG. Emergency department use by the rural elderly. J Emerg Med 2000; 18:289-97.

9. Oliveira EXG, Carvalho MS, Travassos C. Territórios do Sistema Único de Saúde: mapeamento das redes de atenção hospitalar. Cad Saúde Pública 2004; 20:386-402.

10. Carneiro MJ. Apresentação. In: Moreira RJ, organizador. Identidades sociais: ruralidades no Brasil contemporâneo. Rio de Janeiro: DP\&A Editora; 2005. p. 7-13. 
11. Wanderley MNB. A ruralidade no Brasil moderno. In: Giarracca N, organizador. Una nueva realidad en América Latina. Buenos Aires: Consejo Latinoamericano de Ciencias Sociales; 2001. p. 31-44.

12. Instituto Brasileiro de Geografia e Estatística. Acesso e utilização de serviços de saúde - PNAD, 2003. Rio de Janeiro: Instituto Brasileiro de Geografia e Estatística; 2005.

13. Silva PLN, Pessoa DGC, Lila MF. Análise estatística de dados da PNAD: incorporando a estrutura do plano amostral. Ciênc Saúde Coletiva 2002; 7:659 70.

14. Instituto Brasileiro de Geografia e Estatística. Metodologia do censo demográfico 2000. Rio de Janeiro: Instituto Brasileiro de Geografia e Estatística; 2003. (Série Relatórios Metodológicos, 25).

15. Lima-Costa MF, Firmo JOA, Uchoa E. The structure of self-rated health among older adults: the Bambuí health and ageing study (BHAS). Rev Saúde Pública 2004; 38:827-34.

16. Parahyba MI, Veras R, Melzer D. Incapacidade funcional entre as mulheres idosas no Brasil. Rev Saúde Pública 2005; 39:383-91.

17. Instituto Nacional de Câncer. Inquérito domiciliar sobre comportamentos de risco e morbidade referida de doenças e agravos não transmissíveis: Brasil, 15 capitais e Distrito Federal, 2002-2003. Rio de Janeiro: Instituto Nacional de Câncer; 2004.

18. Lee ES, Forthofer RN, Lomimer RJ. Analyzing complex survey data. London: Sage Publications; 1989. (Series: Quantitative Applications in Social Sciences).
19. van Doorslaer E, Masseria C, Koolman X; OECD Health Equity Group. Inequalities in access to medical care by income in developed countries. CMAJ 2006; 174:177-83.

20. Basu J, Mobley LR. Illness severity and propensity to travel along the urban-rural continuum. Health Place 2007; 13:381-99.

21. Robinson J. Age equality in health and social care London: Institute for Public Policy Research; 2002.

22. Thiede M. Information and access to health care: is there a role of trust? Soc Sci Med 2005; 61:1452 62.

23. Suominen-Taipale AL, Martelin T, Koskinen S, Holmen J, Johnsen R. Gender differences in health care use among the elderly population in areas of Norway and Finland. A cross-sectional analysis based on the HUNT study and the FINRISK Senior Survey. BMC Health Serv Res 2006; 6:110.

24. Haugh MR, Morris D, Musil C, Warner C. Older adults' bodily changes and physician contact. Health 1997; 1:81-105.

25. Verbrugge LM, Wingard DL. Sex differentials in health and mortality. Women Health 1987; 12:10345.

26. Mutran E, Ferraro KF. Medical need and use of services among older men and women. J Gerontol 1988; 43:S162-71.

27. Matos DL, Giatti L, Lima-Costa MF. Fatores sócio-demográficos associados ao uso de serviços odontológicos entre idosos brasileiros: um estudo baseado na Pesquisa Nacional por Amostra de Domicílios. Cad Saúde Pública 2004; 20:1290-7.

Recebido em 07/Nov/2006

Versão final reapresentada em 27/Fev/2007

Aprovado em 02/Mar/2007 